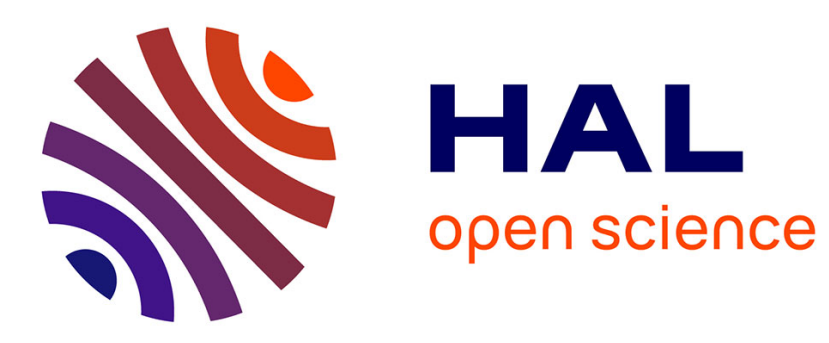

\title{
Atlas-based Quantification of Myocardial Motion Abnormalities: Added-value for the Understanding of CRT Outcome?
}

Nicolas Duchateau, Mathieu de Craene, Gemma Piella, Corné Hoogendoorn, Etelvino Silva, Adelina Doltra, Lluis Mont, María Angeles Castel, Josep Brugada, Marta Sitges, et al.

\section{To cite this version:}

Nicolas Duchateau, Mathieu de Craene, Gemma Piella, Corné Hoogendoorn, Etelvino Silva, et al.. Atlas-based Quantification of Myocardial Motion Abnormalities: Added-value for the Understanding of CRT Outcome?. Statistical Atlases and Computational Models of the Heart (STACOM), MICCAI'10 Workshop, 2010, Beijing, China. pp.65-74, 10.1007/978-3-642-15835-3_7 . hal-02320748

\section{HAL Id: hal-02320748 \\ https://hal.science/hal-02320748}

Submitted on 19 Oct 2019

HAL is a multi-disciplinary open access archive for the deposit and dissemination of scientific research documents, whether they are published or not. The documents may come from teaching and research institutions in France or abroad, or from public or private research centers.
L'archive ouverte pluridisciplinaire HAL, est destinée au dépôt et à la diffusion de documents scientifiques de niveau recherche, publiés ou non, émanant des établissements d'enseignement et de recherche français ou étrangers, des laboratoires publics ou privés. 


\title{
Atlas-based Quantification of Myocardial Motion Abnormalities: Added-value for the Understanding of CRT Outcome?
}

\author{
Nicolas Duchateau ${ }^{1}$, Mathieu De Craene $^{1}$, Gemma Piella ${ }^{1}$, \\ Corné Hoogendoorn ${ }^{1}$, Etelvino Silva ${ }^{2}$, Adelina Doltra ${ }^{2}$, Lluís Mont ${ }^{2}$, \\ $\mathrm{M}^{a}$ Angeles Castel ${ }^{2}$, Josep Brugada ${ }^{2}$, Marta Sitges ${ }^{2}$, and Alejandro F. Frangi ${ }^{1,3}$ \\ 1 CISTIB, Universitat Pompeu Fabra, CIBER-BBN, Barcelona, Spain \\ ${ }^{2}$ Hospital Clínic - IDIBAPS - Universitat de Barcelona, Spain \\ 3 ICREA, Barcelona, Spain
}

\begin{abstract}
In this paper, we present the use of atlas-based indexes of abnormality for the quantification of cardiac resynchronization therapy (CRT) outcome in terms of motion. We build an atlas of normal motion from 21 healthy volunteers to which we compare 88 CRT candidates before and after the therapy. Abnormal motion is quantified locally in time and space using a statistical distance to normality, and changes induced by the therapy are related with clinical measurements of CRT outcome. Results correlate with recent clinical hypothesis about CRT response, namely that the correction of specific mechanisms responsible for cardiac dyssynchrony conditions the response to the therapy.
\end{abstract}

\section{Introduction}

Cardiac resynchronization therapy (CRT) has become one of the main treatments for improving the condition of patients with advanced heart failure 1 . However, around $30 \%$ of the patients implanted with a CRT device fail to clinically respond (around 50\% concerning echocardiographic response) 2]. Measuring mechanical dyssynchrony was recently targeted by a large number of trials, looking for a better selection parameter that could outperform the criteria currently used in clinical practice (symptomatic heart failure with long QRS length and low ejection fraction) 3 .

Single dyssynchrony indexes based on echocardiographic images showed low predictive value, mainly due to their poor reproducibility in a multi-center context 4. In addition, the choice of a single parametric approach discards the complexity of the mechanisms responsible for the dyssynchrony of each patient, which explains such a low predictive value [5. A mechanism-based patient selection strategy has recently been proposed in [6, and contrasts with the previous approaches. The authors first classified patients into specific etiologies of mechanical dyssynchrony, and then studied the response of each class. They concluded that CRT response mainly depends on the ability to correct these specific abnormal mechanisms. Such a strategy also confirmed that understanding the 
patho-physiological mechanisms amenable to CRT may result more optimal for patient selection than arbitrarily relying on the currently established selection criteria.

Despite these conclusions, the classification of patients and the observations made after the therapy largely relied on visual observations, which questions their reproducibility. More novel methods based on myocardial deformation have also been proposed to study abnormal patterns associated with dyssynchrony [7] [8, but their use in a CRT context is also limited by the reproducibility of the measurements. Higher reproducibility can be achieved by accurately synchronizing patient data to a common spatiotemporal reference anatomy. Patient comparison in terms of cardiac motion and deformation was proposed in $[9,[10$ and [1], using the framework of statistical atlases. In particular, an atlas-based pipeline for the quantification of myocardial motion abnormalities has recently been described in [12. This pipeline characterized myocardial velocities of the studied patients according to their distance to normality, in a similar fashion than proposed in 13 for the detection of brain abnormalities. The technique was evaluated in the context of CRT, using a reduced set of patients with one clear specific pattern related to left ventricle (LV) dyssynchrony, namely septal flash (SF) [6].

In this work, we apply this framework to a large database of CRT candidates with a wide spectrum of ventricular dyssynchrony. Our aim is to demonstrate the added value of such an atlas-based distance to normality for the study of response to CRT. We use the local abnormality indexes proposed in 12 to accurately quantify improvements in wall motion induced by CRT. We finally relate the changes in local abnormalities with information about CRT outcome, in order to understand the causes of non-response to the therapy.

\section{Methods}

\subsection{Patient population}

For the present study, data was collected from 21 healthy volunteers, and 88 patients that were candidates for CRT based on current international clinical guidelines (ejection fraction $<35 \%$, QRS duration $>120 \mathrm{~ms}$, and NYHA classification III-IV). The study protocol was approved by our local ethics committee and written informed consent was obtained from all patients. The baseline characteristics for these subjects are summarized in Tab. 1. Clinical response was defined at follow-up, as an increase $\geq 10 \%$ in the 6 min walking test as compared to baseline, or a NYHA functional class reduction $\geq 1$ point, in alive patients without heart transplantation. Echocardiographic response was defined as a reduction $\geq 15 \%$ in the LV end-systolic volume [2], as measured by one experienced observer. Patients who died or had heart transplantation during the study were considered as non-responders.

Echocardiographic (2D US) sequences in a zoomed-in 4-chamber view were acquired for all these subjects, using a commercially available system (Vivid 
This a pre-print version.

The final document is available at http://www.springerlink.com

\begin{tabular}{|c|c|c|c|c|}
\hline & & \multicolumn{2}{|c|}{ CRT candidates } & \multirow[t]{2}{*}{ Volunteers } \\
\hline & & Baseline & Follow-up & \\
\hline Age (years) & & \multicolumn{2}{|c|}{$68 \pm 9$} & $30 \pm 5$ \\
\hline Male gender & & \multicolumn{2}{|c|}{$64(73 \%)$} & $14(67 \%)$ \\
\hline QRS width (ms) & & $178 \pm 29$ & . & $81 \pm 10$ \\
\hline 6 min walking test $(\mathrm{m})$ & & $289 \pm 82$ & $399 \pm 130$ & \\
\hline \multirow[t]{4}{*}{ NYHA funcional class } & I & 0 & $15(17 \%)$ & $21(100 \%)$ \\
\hline & II & $23(26 \%)$ & $49(56 \%)$ & 0 \\
\hline & III & $56(64 \%)$ & $19(22 \%)$ & 0 \\
\hline & IV & $7(8 \%)$ & 0 & 0 \\
\hline \multicolumn{2}{|c|}{ LV end-diastolic volume (mL) } & $247 \pm 88$ & $212 \pm 78$ & $104 \pm 27$ \\
\hline \multicolumn{2}{|c|}{$\mathrm{LV}$ end-systolic volume $(\mathrm{mL})$} & $186 \pm 76$ & $147 \pm 66$ & $41 \pm 9$ \\
\hline \multicolumn{2}{|c|}{ LV ejection fraction (\%) } & $25 \pm 8$ & $33 \pm 9$ & $60 \pm 5$ \\
\hline
\end{tabular}

Table 1: Clinical and echocardiographic characteristics for the population of CRT candidates and for the set of volunteers.

7, General Electric, Milwaukee, WI, USA). The examination was performed at baseline (OFF), 24-72 hours after device implantation $(\mathrm{ON})$ and at follow-up (FU), which corresponded in average to $11 \pm 2$ months after the implant. The sequences were acquired with a breath-hold constraint to minimize the influence of respiratory motion. Resolution was optimized during the acquisition of healthy subjects sequences, and corresponds to an average frame rate of 60 frames/s and a pixel size of $0.24 \times 0.24 \mathrm{~mm}^{2}$. The temporal resolution of the sequences is lower for the set of CRT candidates (around half the frame rate), as they have dilated hearts and therefore require the use of a broader US sector. Their average pixel size is $0.26 \times 0.26 \mathrm{~mm}^{2}$.

The choice of using 2D US and in particular the 4-chamber view was lead by the fact that this view is the one used in clinical routine for the assessment of fast abnormal motion patterns related to LV dyssynchrony, as described in [6]. However, the concepts developed in this paper could readily be applied to 3D US and other imaging modalities once the required temporal resolution is available in standard clinical acquisition protocols. The use of real-time 3D echocardiography 14] 15] is particularly of interest to capture out-of-plane motion, which may increase the accuracy of the proposed analysis, and extend it to specific 3D motion patterns currently not captured by our method, such as torsion.

\subsection{Atlas-based abnormality indexes}

The atlas pipeline described in [12] was applied on the 4-chamber sequences of the two populations described in Sec. 2.1. The choice of a reference among the set of healthy volunteers was addressed using the group-wise normalized mutual information metric proposed in [16, and criteria based on image quality (LV fully visible along the whole sequence, and low heart rate to achieve a higher temporal resolution of the atlas). Statistics on myocardial velocities were computed locally in time and space, at every anatomical location $(\mathbf{x}, t)$ of the reference anatomy. Normal motion was first quantified by computing the average and covariance 


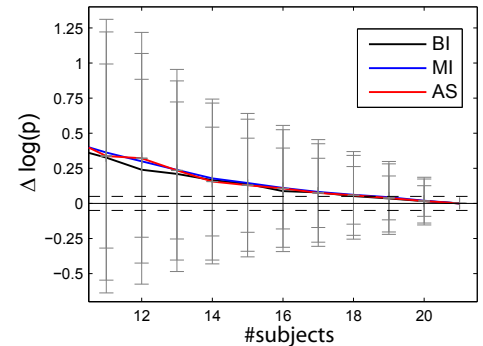

\begin{tabular}{c|ccc} 
Segment & IVC $^{*}$ & Systole $\backslash$ IVC $^{\dagger}$ & Diastole \\
\hline BI & $17.0 \pm 1.6$ & $16.8 \pm 1.2$ & $17.9 \pm 1.3$ \\
MI & $16.7 \pm 1.0$ & $17.1 \pm 1.5$ & $18.0 \pm 1.1$ \\
AS & $17.9 \pm 1.5$ & $19.3 \pm 1.5$ & $19.4 \pm 1.6$
\end{tabular}

* Isovolumic contraction

$\dagger$ Systole excluding the IVC period

Fig. 1: Left: Normalized evolution of the abnormality indexes of one CRT candidate, versus the size of the atlas population. Average over the cardiac cycle and the septal segments (basal inferoseptal [BI], mid inferoseptal [MI], and apical septal [AS]). Error bars represent the standard deviation over 100 random combinations of $N_{s}<N$ subjects. Right: values above which this evolution stabilizes to its final value $\pm 5 \%$ (dashed line), per cardiac segment and temporal window of the cardiac cycle. Average \pm standard deviation values over a set of 14 CRT candidates.

of myocardial velocities for the set of healthy volunteers. Motion abnormalities were then computed for each individual (both volunteers [using leave-one-out cross-correlation] and CRT candidates) by calculating a statistical distance to the velocity distribution of the atlas population (Hotelling's $T$-square statistic). This computation returned a $p$-value at every location $(\mathbf{x}, t)$ of the myocardial wall, low $p$-value meaning high degree of abnormality. As these abnormality maps are defined on a $2 \mathrm{D}+t$ space, one way of visualizing them is to unfold the LV wall around its medial line and to use time as a second dimension. The representation of the $p$-value in this space is similar to M-mode echocardiographic images, classically used to visualize wall motion over time. Examples of this representation are given in Fig. 2, focusing on the septum region.

\section{Relevance of the atlas population}

The computation of a distance to normality assumes that we can trust the atlas population as being representative of normality. In this study, the atlas population has non-dilated hearts, no antecedent of cardiac dysfunction, and its baseline characteristics match with the values found in the literature for a population of patients with normal cardiac function [17. In addition, this population is relatively young (age $30 \pm 5$ years), which means its cardiac function is preserved from lower efficiency raising up when subjects become older.

Number of subjects To justify that the statistics are not biased due to the number of subjects in the atlas population $(N=21)$, we computed the evolution of the abnormality index ( $p$-value) depending on the number of subjects $N_{s}<N$ involved in its computation. This experiment is summarized in Fig. 1 in which the indexes were computed for a reduced set of 14 CRT candidates at each spatiotemporal location $(\mathbf{x}, t)$. These values were normalized towards the value obtained for the largest atlas population, so that the evolution is represented in 


\begin{tabular}{cccc}
$(\%)$ & Segment & SW & LF \\
\hline \multirow{3}{*}{$\mathbf{v}_{\rho}$} & BI & $89.6 \pm 7.4$ & $17.2 \pm 5.0$ \\
& AS & $90.2 \pm 7.4$ & $17.0 \pm 5.3$ \\
& BI & $90.3 \pm 7.3$ & $16.8 \pm 5.0$ \\
\multirow{3}{*}{$\mathbf{v}_{\theta}$} & MI & $90.9 \pm 7.5$ & $15.9 \pm 4.7$ \\
& AS & $91.2 \pm 7.3$ & $15.6 \pm 4.7$ \\
\multicolumn{2}{r}{$\operatorname{randn}(21,10000)$} & $90.2 \pm 7.4$ & $16.7 \pm 5.2$ \\
\hline
\end{tabular}

Table 2: Shapiro-Wilk (SW) and Lilliefors (LF) tests for the distribution of myocardial velocities from 21 healthy volunteers, at each septal segment. Both components of velocities (radial $\mathbf{v}_{\rho}$ and longitudinal $\mathbf{v}_{\theta}$ ) were treated independently. Bottom line: generation of 21 normally distributed random numbers, repeated 10000 times.

the same magnitude scale (\%). The plot on the left represents this evolution for the three septal segments of one CRT candidate. For each value of $N_{s}<N$, the experiment was repeated for 100 random combinations of $N_{s}$ subjects (vertical error bars). In each spatiotemporal region, the number of subjects above which this evolution stabilizes to its final value $\pm 5 \%$ is summarized in the table on the right (average \pm standard deviation over the set of 14 CRT candidates). Based on these values, we can reasonably trust an atlas built with all the available population of subjects (21 volunteers).

Statistical distribution assumptions The $p$-value used for the quantification of motion abnormalities is computed from the Hotelling's $T$-square statistic, under the assumption that the distribution of myocardial velocities over the set of the 21 volunteers is gaussian. We computed the Shapiro-Wilk and the Lilliefors tests at each location $(\mathbf{x}, t)$ to justify this assumption. The results are summarized in Tab. 2, which shows the average values and standard deviation of these tests over the three septal segments, for both components of the velocities treated independently. The last line presents the values of these tests for the generation of 21 normally distributed random numbers, repeated 10000 times. Based on these values, we can reasonably consider that the distribution of velocities is gaussian at each point $(\mathbf{x}, t)$.

\section{Clinical outcome after CRT}

\subsection{Atlas-based quantification of CRT outcome}

We computed $p$-value maps of abnormality for the set of 88 CRT candidates, as described in Sec. 2.2. These maps are illustrated in Fig. 2 at baseline and follow-up, focusing on the septum region for three candidates. The $p$-value is represented in logarithmic scale, and pondered by the sign of radial velocity $\mathbf{v}_{\rho}$ at the same location $(\mathbf{x}, t)$. This mode of representation highlights inward and outward motion of the septum: blue color represents high abnormality with inward motion of the septum, red color being high abnormality for an outward motion. Characteristic abnormality patterns are visible on these three patients 

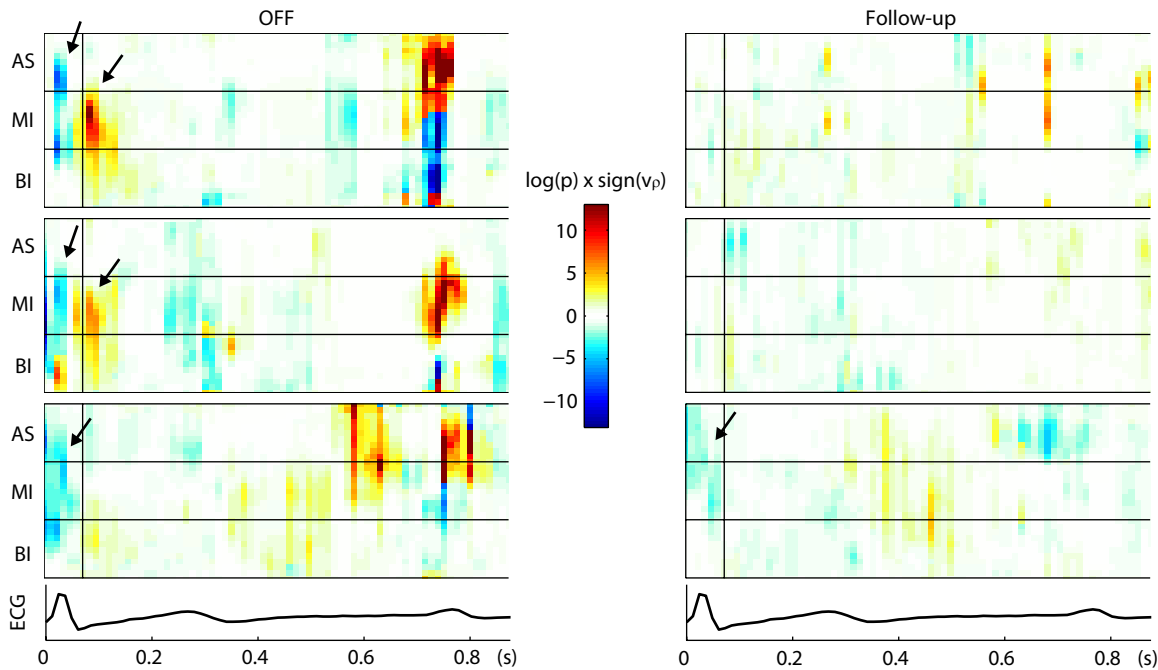

Fig. 2: Abnormality maps in the septal region (vertical axis) for three CRT candidates, at baseline and follow-up: two with SF (top and middle, inward and outward events of SF being indicated by the black arrows), and one with left-right interaction (bottom). The color scale encodes the amount of $p$-value in logarithmic scale, pondered by the sign of the radial velocity. The vertical line indicates the end of the IVC period.

before the therapy, and can be related to specific types of dyssynchrony: the patients at top and middle rows show a fast succession of blue/red color during the IVC period, corresponding to the inward/outward events of SF. This pattern is not visible anymore on the maps at follow-up. In contrast, the bottom row patient has only inward abnormality during the IVC, corresponding to a leftright interaction dyssynchrony. This pattern, despite a slight reduction, is still visible at follow-up.

\subsection{Relation between abnormality reduction and CRT outcome}

The follow-up parameters for the set of CRT candidates are displayed in Tab. 1 . One patient died during the study, and was therefore considered as non-responder. This patient had SF. Among the 87 remaining patients, experimented clinicians visually detected SF at baseline for 58 of them (67\%). This pattern was still present after the therapy for 7 patients $(8 \%)$. The amount of responders and non-responders for these populations is summarized in Tab. 3 .

Regional abnormalities were then compared to CRT outcome for the whole set of CRT candidates, looking for differences between responders and nonresponders. Boxplots in Fig. 3 represent the average of the abnormality indexes over temporal intervals of the cardiac cycle and the three septal segments. The indexes were computed at baseline (OFF), after implant (ON), and at follow-up $(\mathrm{FU})$. Indexes for the atlas population are displayed in gray. In this figure, we mainly observe a reduction of systolic abnormalities (IVC and Systole $\backslash$ IVC), 


$$
\begin{array}{lc|cc}
\multicolumn{1}{c}{} & \multicolumn{2}{c}{\text { Clinical }} \\
\text { Echo } & \text { R } & \text { NR } \\
\cline { 2 - 4 } & \mathrm{R} & 42 & 7 \\
\mathrm{NR} & 30 & 9
\end{array}
$$

88 CRT candidates

$$
\begin{array}{cc|cc}
\multicolumn{1}{c}{} & \multicolumn{2}{c}{\text { Clinical }} \\
\multirow{4}{*}{\text { Echo }} & & \mathrm{R} & \mathrm{NR} \\
\cline { 2 - 4 } & \mathrm{R} & 36 & 5 \\
\mathrm{NR} & 15 & 3
\end{array}
$$

$59 \mathrm{SF}(\mathrm{OFF})$

\author{
Clinical
}

Table 3: Clinical and echocardiographic responders $(R)$ and non-responders (NR) among the set of 88 CRT candidates, the set of 59 patients with SF detected at baseline (58 survived), and the 7 patients for which SF is still present after the therapy.

at basal inferoseptal and mid inferoseptal levels. Responders show a slightly higher reduction of abnormalities at mid inferoseptal level during the IVC. However, abnormality changes before and after CRT are similar for both responders and non-responders at the other spatiotemporal regions, showing that the therapy improves the motion and the coordination of the cardiac chambers in both groups. This also suggests that global computations of abnormality and in general of dyssynchrony cannot distinguish between responders and non-responders. Such a conclusion echoes the clinical scepticism about the limited value of single dyssynchrony indexes for the study of CRT response [4] [5].

As a consequence, we specifically looked at the evolution of abnormalities corresponding to SF, with the underlying objective of confirming the hypothesis that the reduction of specific abnormal patterns may be a better predictor of CRT response. This comparison is illustrated in Fig. 4 for the set of 58 patients who were visually diagnosed as SF at baseline, and for which FU information is available. The displayed points represent the average of the abnormality over the whole septum, and over the temporal windows corresponding to the inward $(\mathcal{I N})$ and outward $(\mathcal{O U} \mathcal{T})$ events of SF, defined as:

$$
\begin{aligned}
\mathcal{I N} & =\left\{t \in I V C \mid \mathbf{v}_{\rho}(t)<0, t<\mathcal{O U} \mathcal{T}\right\} \\
\mathcal{O U} \mathcal{T} & =\left\{t \in I V C \mid \mathbf{v}_{\rho}(t)>0, t>\mathcal{I N}\right\}
\end{aligned}
$$

In this figure, we observe a large reduction of $\mathrm{SF}$ abnormalities for the whole set of responders, confirming that a correction of this specific pattern stands for a good predictor of CRT response. As a comparison, in [6], all the patients for which SF was corrected by CRT were responders.

In our study, there were 7 non-responders among the SF population (12\%), for which a case by case examination helps understanding the reasons for nonresponse: 2 conserved the SF pattern after the therapy (cross symbols on Fig 4); 1 had still highly abnormal dyssynchronous motion, even if SF was resolved; 1 had ambiguous SF at baseline; 2 showed an increase in the 6 min walking test that was not enough to pass the threshold for being considered as clinical responder; and 1 fails to respond without any of these reasons.

Limitations Myocardial velocities were computed under a small displacements hypothesis and assuming stationarity between the acquired frames, as justified in [12. This partially limits artifacts resulting from differences in temporal resolution between the set of healthy volunteers and CRT candidates respectively. 

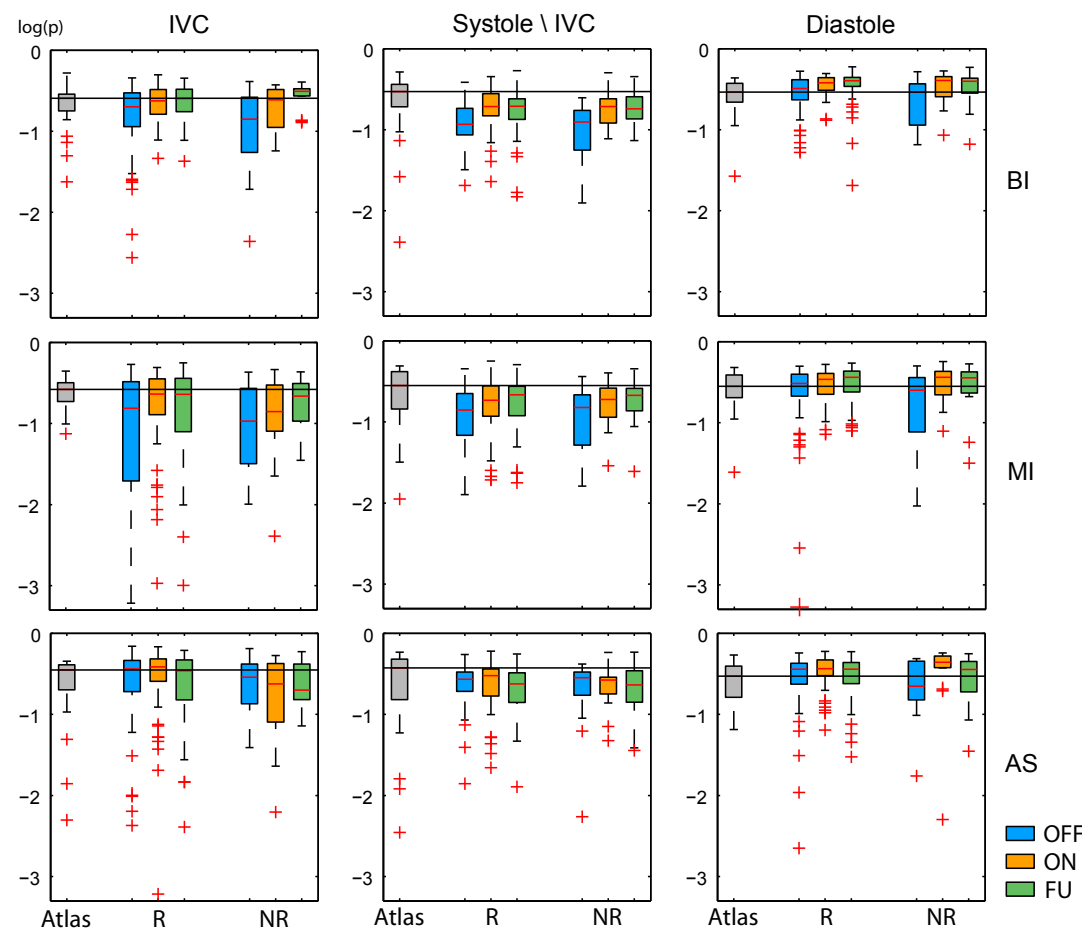

Fig. 3: Comparison of regional abnormality indexes ( $p$-value in logarithmic scale) at baseline (OFF), after implant (ON), and at follow-up (FU). Clustering between responders (R) and non-responders (NR). Average over specific temporal intervals of the cardiac cycle (columns) and septal segments (rows).

The use of $2 \mathrm{D}+\mathrm{t}$ diffeomorphic tracking of the anatomy [15] will be included to our pipeline in further work to reach better accuracy along the continuous timescale.

In this study, results are displayed against clinical response. The observations made with echocardiographic response lead to less conclusions. This can be interpreted by the fact that CRT is able to correct motion abnormalities and therefore improving patient condition (clinical response), without necessarily reversing cardiac remodelling (echocardiographic response). Complementary details discussing the relevance of clinical and echocardiographic response can be found in [2].

\section{Conclusion}

In this paper, we demonstrated the use of atlas-based abnormality indexes for the local quantification of cardiac motion improvements induced by CRT, which is particularly relevant for the understanding of CRT outcome. The conclusions from this work correlate with the hypothesis demonstrated in recent clinical 


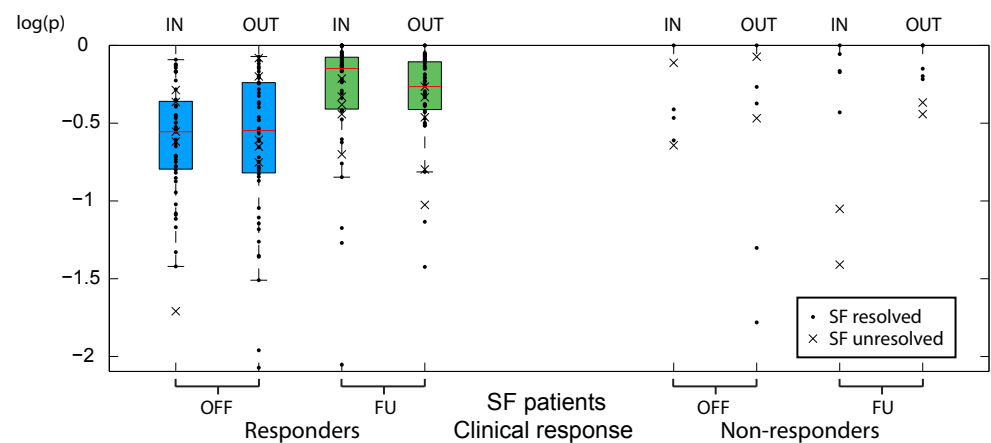

Fig. 4: Comparison of abnormality indexes ( $p$-value in logarithmic scale) for the inward (IN) and outward (OUT) events of SF, at baseline (OFF) and follow-up (FU). Average over the IVC period and the whole septum. Cross symbols indicate patients for which $\mathrm{SF}$ is still observed at $\mathrm{FU}$.

trials, namely that the reduction of specific patterns of dyssynchrony conditions the response to CRT. The prediction of response based on baseline data will be studied in further work, and will include the comparison of individuals to groups of patients for which response is known. Future work will also extend the analysis to strain measurements, as the presence of local infarction might affect CRT response, and is not currently taken into account by indexes focusing on motion only.

Acknowledgments This research has been partially funded by the Industrial and Technological Development Center (CDTI) under the CENIT-CDTEAM and CENITcvREMOD programs and by the European Commission's project euHeart (FP7-ICT224495). Gemma Piella was supported by the Ramón y Cajal Programme from the Spanish Ministry of Science and Innovation. Adelina Doltra was supported by a PostResidency Award from Fundació Clínic.

\section{References}

1. M. G. St John Sutton, T. Plappert, W. T. Abraham, A. L. Smith, D. B. DeLurgio, A. R. Leon, E. Loh, D. Z. Kocovic, W. G. Fisher, M. Ellestad, J. Messenger, K. Kruger, K. E. Hilpisch, M. R. S. Hill, and for the MIRACLE Study Group, "Effect of cardiac resynchronization therapy on left ventricular size and function in chronic heart failure," Circulation, vol. 107, no. 15, pp. 1985-1990, 2003.

2. G. B. Bleeker, J. J. Bax, J. W.-H. Fung, E. E. van der Wall, Q. Zhang, M. J. Schalij, J. Y.-S. Chan, and C.-M. Yu, "Clinical versus echocardiographic parameters to assess response to cardiac resynchronization therapy," American Journal of Cardiology, vol. 97, no. 2, pp. 260 - 263, 2006.

3. J. J. Bax, T. Abraham, S. S. Barold, O. A. Breithardt, J. W. Fung, S. Garrigue, I. Gorcsan, John, D. L. Hayes, D. A. Kass, J. Knuuti, C. Leclercq, C. Linde, D. B. Mark, M. J. Monaghan, P. Nihoyannopoulos, M. J. Schalij, C. Stellbrink, and C.-M. Yu, "Cardiac Resynchronization Therapy: Part 1-Issues Before Device Implantation," Journal of the American College of Cardiology, vol. 46, no. 12, pp. 2153-2167, 2005. 
4. J.-U. Voigt, "Rocking will tell it," European Heart Journal, vol. 30, no. 8, pp. 885886,2009

5. B. K. Fornwalt, J. G. Delfino, W. W. Sprague, and J. N. Oshinski, "It's time for a paradigm shift in the quantitative evaluation of left ventricular dyssynchrony," Journal of the American Society of Echocardiography, vol. 22, no. 6, pp. 672-676, 2009.

6. C. Parsai, B. H. Bijnens, G. R. Sutherland, A. Baltabaeva, P. Claus, M. Marciniak, V. Paul, M. Scheffer, E. Donal, G. Derumeaux, and L. Anderson, "Toward understanding response to cardiac resynchronization therapy: left ventricular dyssynchrony is only one of multiple mechanisms," European Heart Journal, vol. 30, no. 8, pp. 940-949, 2009.

7. V. Delgado, C. Ypenburg, R. J. Van Bommel, L. F. Tops, S. A. Mollema, N. A. Marsan, G. B. Bleeker, M. J. Schalij, and J. J. Bax, "Assessment of left ventricular dyssynchrony by speckle tracking strain imaging: Comparison between longitudinal, circumferential, and radial strain in cardiac resynchronization therapy," Journal of the American College of Cardiology, vol. 51, no. 20, pp. 1944-1952, 2008.

8. A. K. Rutz, R. Manka, S. Kozerke, S. Roas, P. Boesiger, and J. Schwitter, "Left ventricular dyssynchrony in patients with left bundle branch block and patients after myocardial infarction: integration of mechanics and viability by cardiac magnetic resonance," European Heart Journal, vol. 30, no. 17, pp. 2117-2127, 2009.

9. A. Rao, R. Chandrashekara, G. I. Sanchez-Ortiz, R. Mohiaddin, P. Aljabar, J. V. Hajnal, B. K. Puri, and D. Rueckert, "Spatial transformation of motion and deformation fields using nonrigid registration," IEEE Transactions on Medical Imaging, vol. 23, no. 9, pp. 1065-1076, 2004.

10. R. Chandrashekara, R. Mohiaddin, and D. Rueckert, "Comparison of cardiac motion fields from tagged and untagged MR images using nonrigid registration," in Proc. FIMH, LNCS 3504, pp. 425-433, 2005.

11. N. F. Rougon, C. Petitjean, and F. J. Preteux, "Building and using a statistical 3D motion atlas for analyzing myocardial contraction in MRI," in Proc. SPIE Medical Imaging 5370, pp. 253-264, 2004.

12. N. Duchateau, M. De Craene, E. Silva, M. Sitges, B. H. Bijnens, and A. F. Frangi, "Septal flash assessment on CRT candidates based on statistical atlases of motion," in Proc. MICCAI, LNCS 5762, pp. 759-766, 2009.

13. O. Commowick, P. Fillard, O. Clatz, and S. K. Warfield, "Detection of DTI white matter abnormalities in multiple sclerosis patients," in Proc. MICCAI, LNCS 5241, pp. $975-982,2008$.

14. O. I. Soliman, M. L. Geleijnse, D. A. Theuns, B. M. van Dalen, W. B. Vletter, L. J. Jordaens, A. K. Metawei, A. M. Al-Amin, and F. J. ten Cate, "Usefulness of left ventricular systolic dyssynchrony by real-time three-dimensional echocardiography to predict long-term response to cardiac resynchronization therapy," American Journal of Cardiology, vol. 103, no. 11, pp. 1586-1591, 2009.

15. M. De Craene, G. Piella, N. Duchateau, E. Silva, A. Doltra, J. D'Hooge, O. Camara, M. Sitges, and A. F. Frangi, "Temporal diffeomorphic free-form deformation for strain quantification in 3D-US images," in Proc. MICCAI, LNCS, 2010. to appear.

16. C. Hoogendoorn, T. Whitmarsh, N. Duchateau, F. M. Sukno, M. De Craene, and A. F. Frangi, "A groupwise mutual information metric for cost efficient selection of a suitable reference in cardiac computational atlas construction," in Proc. SPIE Medical Imaging, vol. 7623, p. 76231R, 2010.

17. H. Feigenbaum, Echocardiography, ch. Echocardiographic measurements and normal values, pp. 658-695. Philadelphia: Lea and Febiger, 1994. 